\title{
Blockchain-based Supply Chain Traceability for COVID-19 PPE
}

This paper was downloaded from TechRxiv (https://www.techrxiv.org).

\section{LICENSE}

CC BY 4.0

SUBMISSION DATE / POSTED DATE

$12-11-2020 / 12-11-2020$

\section{CITATION}

Omar, Ilhaam; Debe, Mazin; Jayaraman, Raja; Salah, Khaled; Omar, Mohammed; Arshad, Junaid (2020): Blockchain-based Supply Chain Traceability for COVID-19 PPE. TechRxiv. Preprint.

https://doi.org/10.36227/techrxiv.13227623.v1

$\mathrm{DOI}$

10.36227/techrxiv.13227623.v1 


\section{RESEARCH}

\section{Blockchain-based Supply Chain Traceability for COVID-19 PPE}

\author{
Ilhaam Omar ${ }^{1}$ \\ , Mazin Debe ${ }^{2}$ \\ , Raja Jayaraman ${ }^{1 *}$ \\ , Khaled Salah ${ }^{2}$ \\ , Mohammed Omar ${ }^{1}$ \\ and Junaid Arshad ${ }^{3}$
}

\begin{abstract}
The COVID-19 pandemic has severely impacted many industries, in particular the healthcare sector exposing systemic vulnerabilities in emergency preparedness, risk mitigation, and supply chain management. A major challenge during the pandemic was related to the increased demand of Personal Protective Equipment (PPE) resulting in critical shortages for healthcare and frontline workers. The lack of information visibility combined with the inability to precisely track product movement within the supply chain requires an robust traceability solution. Blockchain technology is a distributed ledger that ensures a transparent, safe, and secure exchange of data among supply chain stakeholders. The advantages of adopting blockchain technology to manage and track PPE products in the supply chain include decentralized control, security, traceability, and auditable time-stamped transactions. In this paper, we present a blockchain-based approach using smart contracts to transform PPE supply chain operations. We propose a generic framework using Ethereum smart contracts and decentralized storage systems to automate the processes and information exchange and present detailed algorithms that capture the interactions among supply chain stakeholders. The smart contract code was developed and tested in Remix environment, and the code is made publicly available on Github ${ }^{[1]}$. We present detailed cost and security analysis incurred by the stakeholders in the supply chain. Adopting a blockchain-based solution for PPE supply chains is economically viable and provides a streamlined, secure, trusted, and transparent mode of communication among various stakeholders.
\end{abstract}

Keywords: Ethereum; Cybersecurity; PPE Supply Chain; Traceability; COVID-19

\section{Introduction}

The sudden and rapid spread of COVID-19 pandemic has affected on people's lives and communities across the world. The impact of COVID-19 is not only limited to healthcare but has also affected finance, trade, and global economy and other sectors. Asian Development Bank estimated that the cost of the pandemic could range between $\$ 2$ trillion and $\$ 4.1$ trillion globally [1]. One of the critical challenges during COVID-19 pandemic has been the timely and sufficient availability of Personal Protective Equipment (PPE) prioritising front-line workers and medical staff.

${ }^{[1]}$ https://github.com/MazenDB/TrackingPPE 
However, the COVID-19 highlighted major shortcomings in the current healthcare supply chains to meet the extraordinary demand for PPE. Factors such as hoarding, panic buying, and misuse of PPE has immensely disrupted the supply chain putting many lives at risk [2]. The sudden increase in demand for PPE, such as gloves, gowns, face shield, surgical masks, and goggles has triggered overwhelming global production and corresponding price increase making inequitable distribution of access to PPE. During COVID-19 pandemic, healthcare facilities (clinics and hospitals) faced extraordinary demand to provide necessary care to patients around the clock. Healthcare workers on the front-line are at a higher risk being exposed to the virus. Hence, healthcare workers must wear appropriate PPE not only to prevent straining the healthcare system but also to effectively reduce further disease transmission. However, stockpiles of PPE in various countries are diminishing due to the pandemic's effect, has caused clinics and hospitals to repeatedly use single-use items. However, this crisis is not new to the world as supply chain vulnerabilities were confronted during the year 2014 Ebola virus and year 2009 H1NI1 influenza epidemics [3]. It is observed that the most significant challenge during a crisis is to ensure that an adequate quantity of PPE items are available for healthcare workers to use as and when needed [2]. Consequently, combating this challenge requires creating an traceable, efficient PPE supply chain system.

An efficient supply chain is fundamental to a fully-functional healthcare system. Thus, the health supply system must be designed to quickly and reliably deliver crucial health commodities such as medicines, vaccines, and PPE during infectious disease outbreaks. The COVID-19 outbreak has further exacerbated supply chain vulnerabilities across different industries as a result of travel bans and factory shutdowns. This added newer challenges to PPE supply chains, as many countries rely on exporting PPE rather than stockpiling to optimise use of resources. Moreover, there has been a rise in counterfeit PPE amid the COVID-19 outbreak [4]. For example, in India, around 50,000 out of 170,000 PPE kits failed the safety and quality test and had to be destroyed [5].

Blockchain technology offers a distributed, secure and transparent approach to information exchange in the supply chain. Blockchain technology continues to prove its capabilities in various areas in the healthcare sector, such as managing patient healthcare records, simplifying clinical trial processes, tracking donations, and supplies. This is possible due to the advancement in blockchain technology since its first release in the 2008 in the form of Bitcoin. However, with Ethereum, representing the second-generation blockchain technology [6], the use of blockchain in diverse setting has grown especially due to introduction of smart contracts, which act as self-managing software in the network. Hence, blockchain can facilitate secure information exchanges among geographically dispersed stakeholders, improve global trade logistics such as transportation management, procurement, tracking and tracing shipments, and trade finance. In this paper we integrate Ethereum smart contracts along with decentralized storage technology to propose a blockchain solution for tracking PPE from its manufacturing stage to its end users. Our solution requires both on-chain and off-chain storage to record information. The on chain records in the form of transactions are stored within the ledger whereas the off chain storage is used by smart contracts for documentation and verification purposes. 
The primary objective of this paper is to develop a blockchain-based, trusted PPE tracking system. The main contributions of are summarized as follows:

- We develop an Ethereum blockchain-based tracking system to track and verify PPE via secure and trusted distributed ledger on the blockchain network using smart contracts and decentralized storage.

- We present a framework along with the algorithms that define the working principles of the proposed blockchain-based PPE tracking system and detailed sequence diagram detailing stakeholder interactions.

- We test and validate various scenarios of the proposed system functions to assess the practicality of the proposed solution.

- We present cost and security analysis to demonstrate the feasibility of implementing the proposed solution.

The remainder of this paper is organized as follows: Section 2 provides a brief background on the PPE supply chain and explains the significance of utilizing blockchain solution for tracking. Section 3 provides related literature. Section 4 presents the system methodology and architecture of the proposed system, the implementation details are discussed in Section 5. Section 6 presents the results of testing the proposed solution. Furthermore, Section 7 details the cost and security analysis of the proposed solution, and Section 8 presents the conclusions.

\section{Background}

In this section, we provide background information related to the current PPE supply chain challenges, and explain the importance of adopting blockchain technology in enhancing the tracking and traceability of PPE.

\subsection{PPE Supply Chain Challenges}

A supply chain system comprises various elements, including people, organizations, resources, and activities such as management and planning. These elements work together in moving a product or service from the manufacturer to the end-user, involves collaboration and coordination with various stakeholders such as suppliers, shippers, distributors, and wholesalers. A typical PPE supply chain shares similarities with this model and is illustrated in fig. 1. However, crucial supply chain elements have been disrupted in the PPE supply chain owing to the COVID-19 pandemic. For instance, manufacturers encountered difficulty in meeting the sudden surge in PPE demand due to bottlenecks in the process and material shortage.

However, the lack of transparency and asymmetric information among multiple entities are major challenges in the healthcare supply chain. Each entity in the process manages and controls their data in silos that limits the view of other stakeholders in the chain affecting lead times and overall efficiency of the business operation. This potentially results in delays due to poor coordination and collaboration. Moreover, it may give rise to discrepancies in records as each stakeholder only trusts data owned by them without consensus with other stakeholders in the supply chain process resulting in intentional and unintentional data inconsistencies.

Finally, as with any other natural catastrophe, the COVID-19 pandemic also witnessed opportunistic businesses taking advantage of the chaotic management to produce counterfeits. This problem is aggravated due to sudden heavy reliance of 


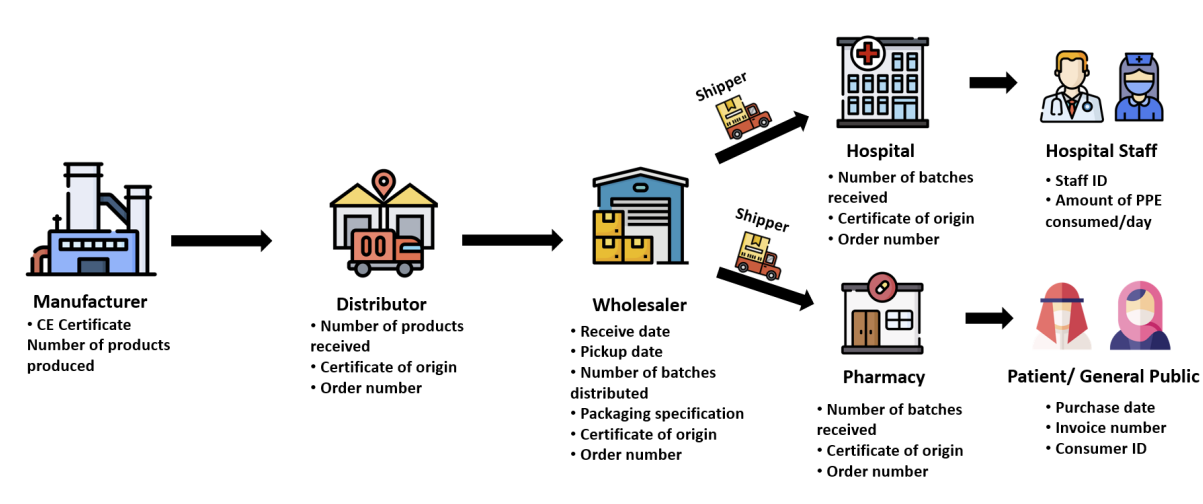

Figure 1 PPE supply chain process flow

customers on online marketplaces which has historically been low-barrier for fake and substandard products [7]. For instance, it has been reported that Amazon had to remove more than 1 million fake products that claimed to protect consumers from the COVID-19 [8].

\subsection{Enhancing PPE supply chain using blockchain technology}

The adverse impact of COVID-19 pandemic is not limited to local supply chains alone but has also affected international trade as well. The flow of goods and services has been disrupted due to trade restrictions, which resulted in logistic partners delaying transportation and suppliers halting production. Hence, technologies such as blockchain and decentralized storage systems are being studied in trade networks. Blockchain has the potential to enable supply chain processes to become more tolerant during future unanticipated outbreaks. It is a sequence of blocks chained together using cryptography technology in which each block holds records of verified and valid transactions that cannot be modified or deleted by anyone in the network, unlike conventional public ledgers [9]. The ledger is distributed to all nodes in the network, which in turn promotes transparency and minimizes the chances of data manipulation [9].

Blockchain can be used to satisfy the requirements of the PPE supply chain system, such as monitoring PPE supply, distribution, and consumption to predict potential distribution needs or supply pitfalls during outbreaks in an efficient manner [10]. It can monitor proficiently due to its inherent characteristics such as immutability and decentralization. For instance, adopting a decentralized network in the supply chain would allow stakeholders to become aware of all the activities taking place without interference from third-parties. Moreover, ensuring immutability reassures stakeholders that unauthorized users will not be able to tamper the records stored in the distributed network due to the cryptographic nature of the technology and time-stamped records [9]. These features are crucial in carefully monitoring PPE distribution and purchase to ensure that patients receive the best healthcare available during epidemics while making sure that the PPE available in the market complies with the standards set by regulatory authorities such as the Centers for Disease Control and Prevention. 
Moreover, the distributed nature of the blockchain network promotes information sharing simultaneously as each member of the network would have a copy of the ledger, containing all the transactions that occur in the supply chain. This feature is beneficial as stakeholders would be able to make decisions much faster as they would be made aware of all the activities occurring in real-time. Hence, transparently sharing knowledge maximizes opportunities to increase overall PPE preparedness. This may include sharing information such as the estimated number of PPE needed in each clinic and hospital or finding means to prioritize limited resources based on the orders placed by the government.

\section{RELATED WORK}

In this section, we discuss the relevant literature on the application of blockchain technology in the supply chain industry. Toyoda et al. used blockchain technology in conjunction with radio frequency identification (RFID) technology to detect and track counterfeit products. The use of blockchain in this case especially protects against cloning attacks for traditional RFID-based approaches. The authors proposed a novel product ownership management system (POMS) of RFID-attached products that utilize the Ethereum platform to prove product ownership [11]. On the other hand, Malik et al. introduced ProductChain to support provenance in supply chain products. The paper proposes the use of a three-tiered architecture that ensures data availability while limiting access to competitive partners and handling valid transactions [12]. Similarly, Wang et al. proposed a smart contract-based solution to trace products in the supply chain to register products and allowing users to track them via events triggered in the contract [13].

However, limited research has been conducted with respect to the application of blockchain within healthcare supply chain system in general and their use for PPE supply chains in particular. We believe that the COVID-19 pandemic has highlighted the need for improved transparency and efficiency within these supply chains. Therefore, we propose a solution tailored specifically towards the PPE supply chain so as to enhance the visibility of stakeholders across the supply chain and enhance efficiency of business processes. Details of our proposed solution along with validation and, cost and security analysis is presented in the following sections.

\section{PROPOSED BLOCKCHAIN-BASED PPE TRACKING SOLUTION}

We propose a blockchain-based solution for effective tracking of PPE to ensure endto-end traceability while maintaining quality assurance so as to ensure end-users that items being sold to them comply with the regulatory standards. Our solution adopts blockchain technology to promote transparency between various stakeholders, including manufacturers, distributors, shippers, wholesalers, and providers such as hospitals and clinics in the PPE supply chain. Moreover, our system develops smart contracts and decentralized storage technology using the Ethereum network, as demonstrated in fig. 2. This is to enable authorized users in the network to track orders in real-time by using the unique product ID allotted to each PPE item. 


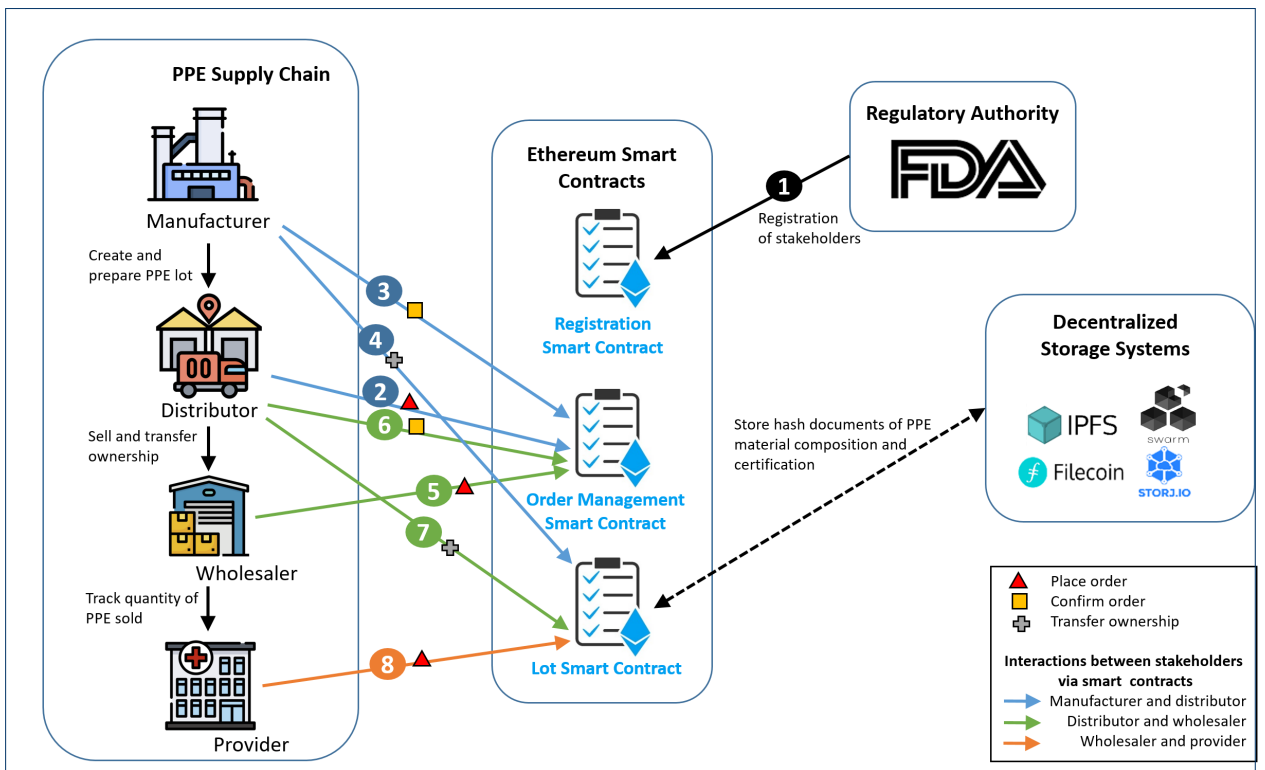

Figure 2 System overview of a blockchain-based PPE tracking process using Ethereum smart contracts and decentralized storage system.

\subsection{Stakeholders and components}

We describe the role of each stakeholder and component in the proposed system below.

- Regulatory Authority: Responsible for registering stakeholders in the blockchain network as well as monitoring the overall supply chain process. Also, it ensures that any PPE, medical equipment, or drugs available in the country abide by the necessary rules and regulations set by authorities such as the US Food and Drug Administration to ensure their safety, quality, and efficacy.

- Manufacturer: Creates products such as pharmaceutical or medical supplies using raw materials found in research labs and manufacturing plants. A manufacturer also studies the market, identifies the needed products, acquires the raw materials, determines the sufficient quantity required to meet customer demand while making sure these products satisfy regulatory standards. Simultaneously, a manufacturer must be able to predict events that would result in potential PPE shortages, such as raw material disruptions during natural disasters or production disruption during pandemics.

- Distributor: Obtains the medical supplies and equipment from manufacturers and distributes them to wholesalers or delivers them to healthcare facilities or clinics. During the process, the distributor may repackage or re-label to ensure special care is taken when handling medical products. Distributors act as an intermediary entity between the manufacturer and providers in the supply chain.

- Wholesaler: The role of the wholesaler is to mainly satisfy the needs of the healthcare provider, making PPE available in large quantities.

- Shipper: When orders are confirmed, wholesalers communicate with the shipment company to provide transportation service in return for a shipping fee 
that would be agreed by both the wholesaler and the healthcare provider. When the shipment is confirmed, the shipper provides real-time status of the order in the network for both parties to see.

- Hospital/Pharmacy: Healthcare providers vary as these can be, clinics, hospitals, pharmacies, etc. They are responsible for supplying healthcare equipment to their staff and consumers. One of their key responsibilities is to monitor the quantity of PPE required while providing the appropriate demand information to distributors or wholesalers to help identify potential distribution challenges or supply shortages.

- Consumers: These can be hospital staff, patients, or even the general public. They influence the demand for PPE items in the supply chain and usually only engage with the healthcare providers.

- Ethereum smart contracts: A code generated to automatically execute functions according to the terms and conditions agreed upon by stakeholders in the network. It acts as a software agent which verifies transactions without interference from third parties. Our proposed solution comprises of three smart contracts where each one is specialized in a particular task. The registration contract focuses on registering all the stakeholders in the network while the order management contract handles and manages the orders. Lastly, the lot contract details the description of the lot in terms of the quantity it holds, the type of PPE, certification etc. [14].

- Decentralized storage technology: Using a peer-to-peer distributed file system such as Filecoin or Interplanetary File System (IPFS) aid in connecting all nodes in the network to the same file system [15]. Authorized users in the network can store large amounts of data in IPFS and store the immutable IPFS links in the blockchain transactions, which are time-stamped and secured using cryptographic technology. This is beneficial as users do not have to store the data on the chain. Thus, the combination of blockchain and distributed storage proves to be valuable since the nodes in the network would only store the indexed information, which would help locate where the data is stored.

\subsection{Process Flow}

The system is captured as a series of functions and events in sequence diagrams 3,4 and 5 highlighting the interaction between each stakeholder and contracts. Initially, each stakeholder in the network is registered using their Ethereum address in the registration contract. The system then allows the distributor to deploy the OrderManager contract and upload information related to the PPE such as Product ID, quantity required, manufacturer address by calling the placeOrder() function as shown in Fig.3. This triggers an event to all the stakeholders that the order has been placed successfully. The manufacturer uses this information to upload the order ID and status of the order using the confirmOrder() function. Then an event is triggered depending on the status of the order alerting the stakeholders, whether it is accepted or rejected.

The manufacturer then deploys the Lot contract when the order is accepted. Then details such as the batch number, product name, and ID along with the quantity are uploaded in the Lot contract, and files such as product certification and material 


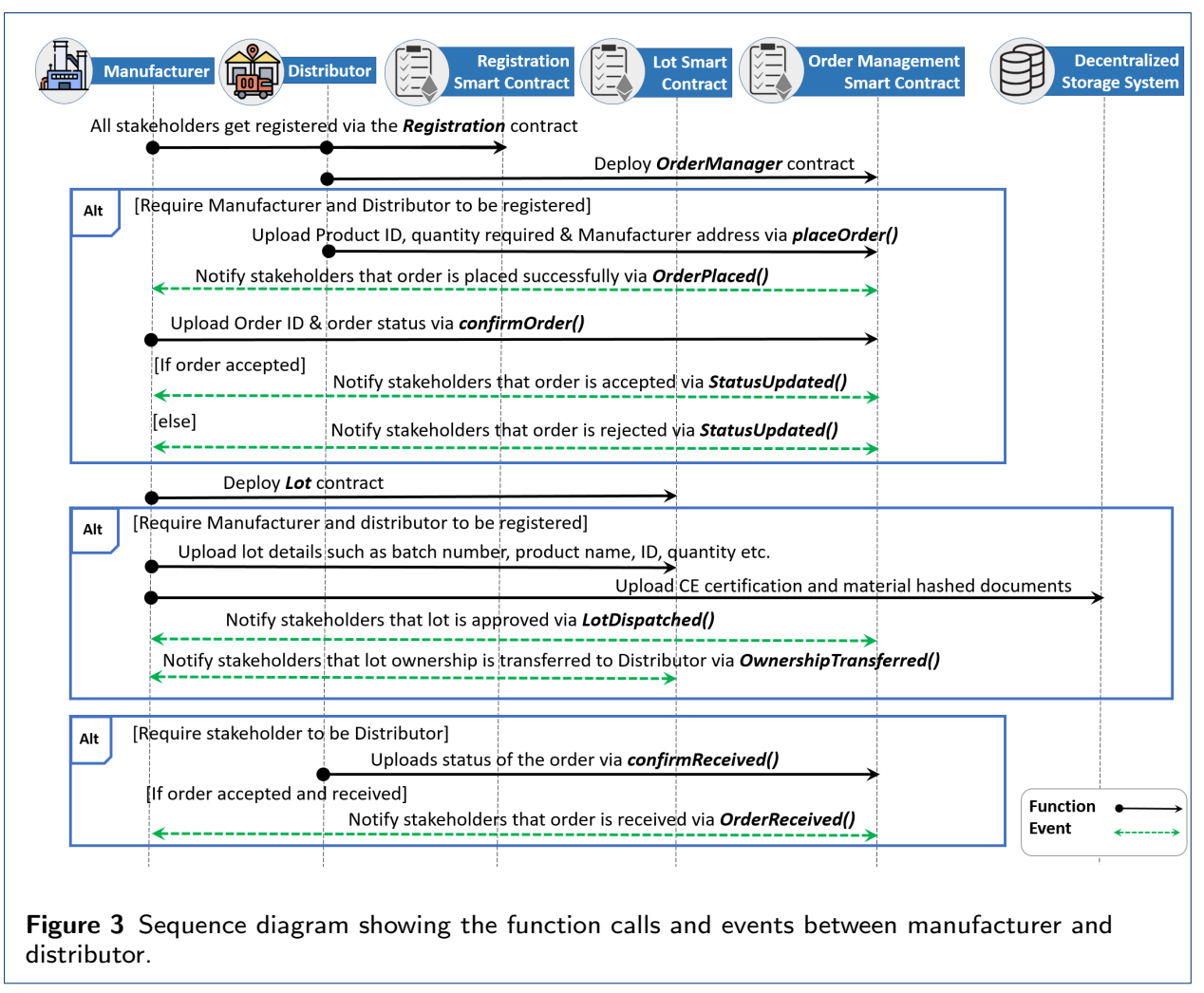

composition are uploaded as hash documents in the decentralized storage system such as IPFS. An event is then triggered, alerting the stakeholders that the lot is approved and ownership is transferred to the distributor. When the order is received by the distributor, then the distributor uploads the status of the order using the confirmReceived() function. Finally, the stakeholders are notified that it is received only when the order is delivered successfully and accepted by the distributor.

However, Fig. 4 captures the interactions between a distributor and wholesaler. Initially, the distributor uploads the information required to place the order, such as the quantity and product ID, using the placeOrder() function. This alerts the stakeholders in the network that the order is placed successfully, leading to order confirmation by the distributor using the confirmOrder() function. Furthermore, the order status is notified to all stakeholders similar to the interaction between the manufacturer and distributor. The ownership is transferred from the distributor to the wholesaler upon successful order completion. Finally, an alert is triggered to all stakeholders when the order is received and accepted.

Moreover, we also capture the interactions between the wholesaler and provider, such as a hospital or pharmacy, as illustrated in fig .5. In this case, the wholesaler specifies the quantity required by the provider by calling the sellToProvider() function. The contract then updates the remaining quantity available in the lot, and when the quantity reaches zero, the contract self destructs.

\section{IMPLEMENTATION}

This section presents the implementation details of the blockchain-based solution for enhancing the traceability and managing PPE. The system overview presented 


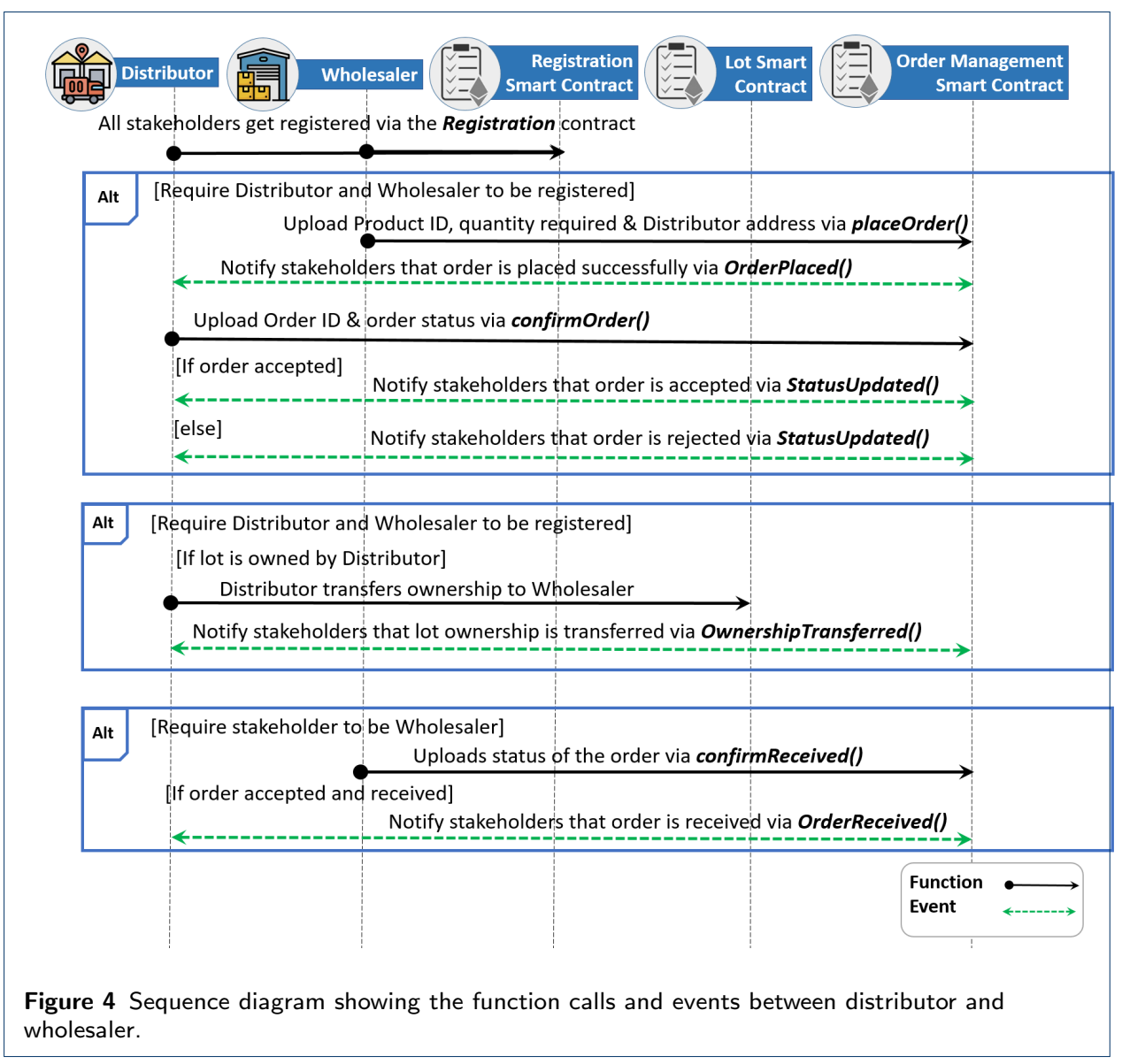

previously was implemented on a test Ethereum blockchain. Remix IDE was exploited to deploy smart contracts using Solidity language.

There are three different types of smart contracts in our solution: Registration smart contract, Lot smart contract, and the order management smart contract. These smart contracts are used for different purposes explained below.

- Registration smart contract: This smart contract is deployed by the owner of the system, such as a government or other entity. The registration smart contract is deployed once and used to manage the addresses of all stakeholders. Each stakeholder is identified by a unique identifier made up of 20 bytes called the Ethereum Address (EA). Manufacturers, distributors, wholesalers, and providers are all registered in this smart contract. In addition, other related smart contracts consult it to verify the identity of an entity through its EA.

- Order management smart contract: Registered wholesalers contact distributors to place an order for specific PPE. Distributors can also order a new PPE lot from manufacturers if they are out of stock. These processes are done through this smart contract, where it announces the placement of new orders. The smart contract verifies the identity of the participating entities before placing the order. Subsequently, all updates about the orders are recorded in this smart contract, including the order confirmation and order delivery. Each order here is recognized by a unique 32 -byte number. This identifier is 


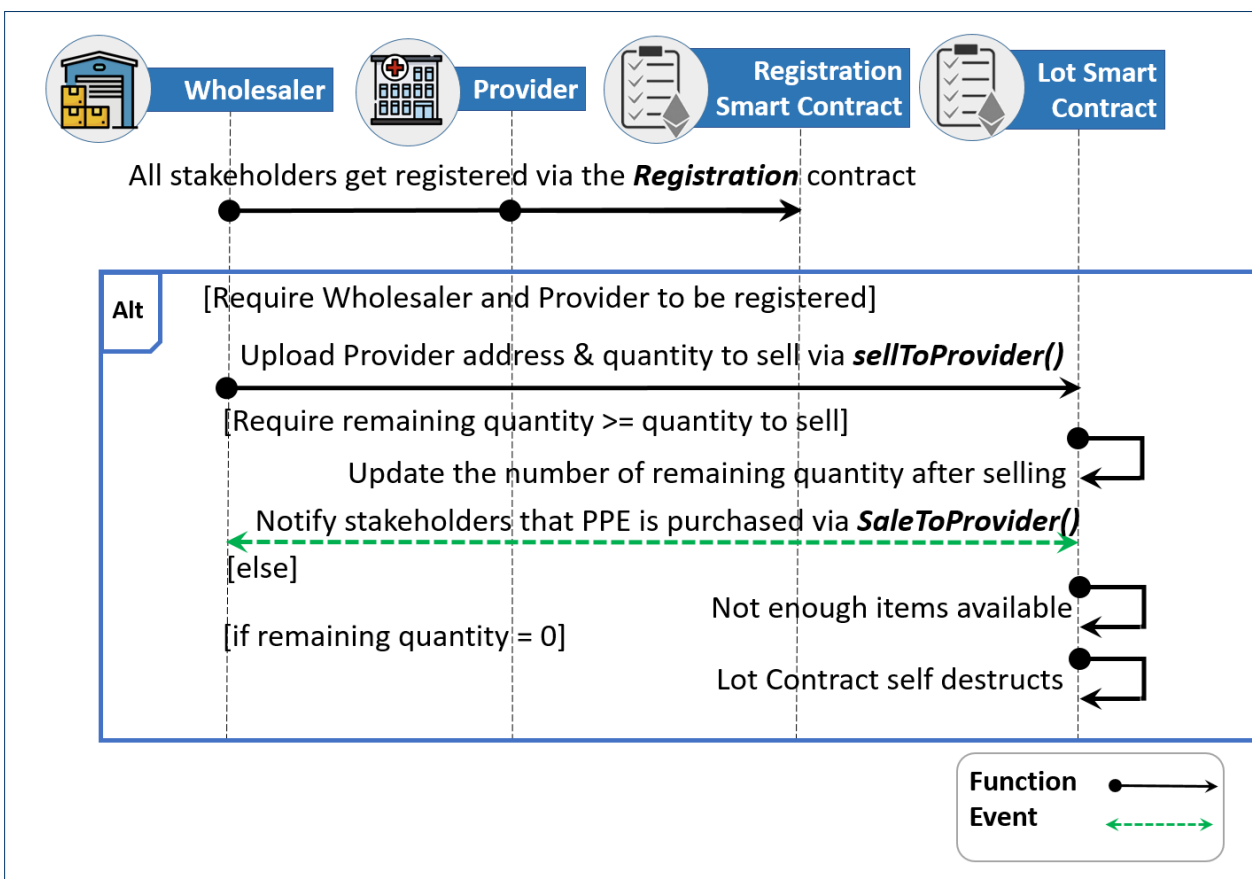

Figure 5 Sequence diagram showing the function calls and events between wholesaler and provider.

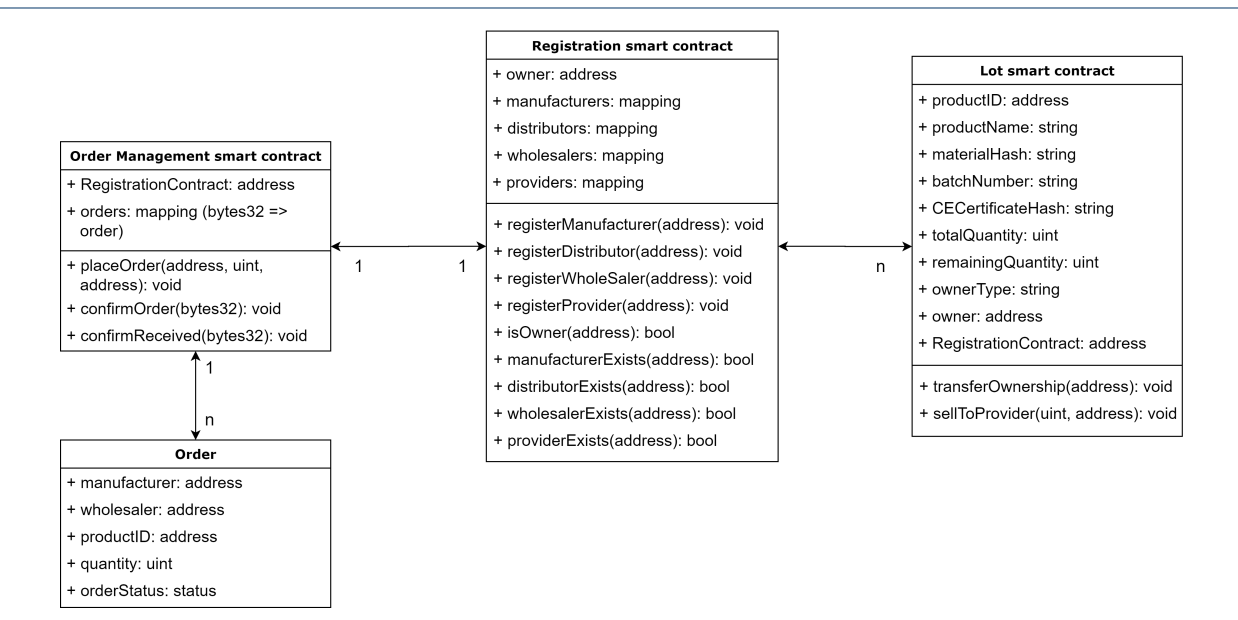

Figure 6 Relationship between different entities of the system.

mapped to the product ID, the quantity ordered, the address of the entity who placed the order, and the address of the order receiver.

- Lot smart contracts: These smart contracts are deployed whenever a new lot of PPE is dispatched. Each lot smart contract is identified by its EA, and the details about the lot are all stored in the contract once it is created. These include the type of product in the lot, its name, the material and composition hash file, the batch number, the $\mathrm{CE}$ certificate hash file, the total quantity originally ordered, and the remaining quantity after distributing it to various providers. In addition, the lot smart contract keeps track of the ownership 
of the PPE lot. Any change of ownership from manufacturing to usage is recorded on the blockchain with the owner's EA address and type. The fixed EA address of the registration smart contract is also stored for reference to validate the identity of wholesalers.

Fig. 6 shows the different entities of the solution and interactions among them. The registration smart contract registers the addresses of all stakeholders and stores them. This smart contract is referred to by other contracts to verify the identity of a specific entity. For instance, the lot smart contract checks the EA of the entity requesting a transfer of ownership for a lot as well as the EA of the new owner. Each new shipped lot has a corresponding lot smart contract that stores the details of the lot. This type of smart contracts can only be deployed by registered manufacturers. An order for the lot should be placed prior to its production. This is achieved through the order management smart contract. Wholesalers and distributors can place orders, and the smart contract informs the appropriate entities and records the request. Subsequently, the manufacturer or the distributor confirms the order by contacting the smart contract. Lastly, the order requester confirms the delivery of the shipment by triggering the relevant method in the smart contract. The order in this smart contract comprises several details, as shown in the figure 6 . It records the address of the entity that requested the order, the entity that confirmed it, the type of product, and the quantity ordered. Additionally, further details about the time of requesting, confirmation, and order delivery can be deduced from the events broadcasted at each step.

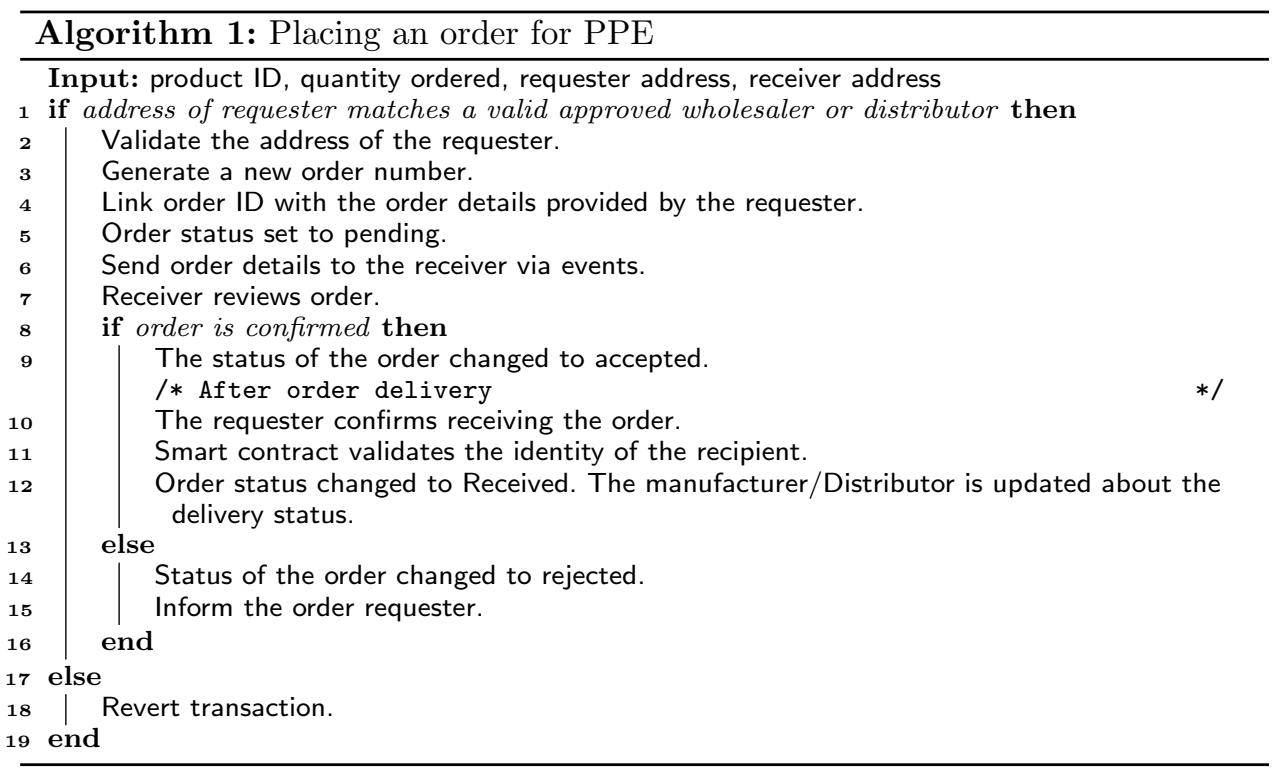

The process of acquiring a PPE lot by a wholesaler starts by sending an order request to the manufacturer. Algorithm 1 presents the process of placing an order for a PPE lot. The wholesaler triggers the method for placing an order in the order management smart contract with the parameters set to the specific product wanted and the quantity required. If the distributor does not have the specific items requested by the wholesaler, it can request the transfer of a new lot by the 
manufacturer. The smart contract validates the addresses of the requester as well as the entity that the products are requested from. After validation, a new 32-byte order number is generated by the contract to refer to this specific order. The order is sent to the order receiver that reviews it and responds to the order request. The request can be rejected if the product ID is invalid, the quantity is unavailable, or for any other reason. On the other hand, if said order is valid, the receiver accepts it, changing its status in the smart contract. The requester of the PPE order makes the final modification in the order status when it is received.

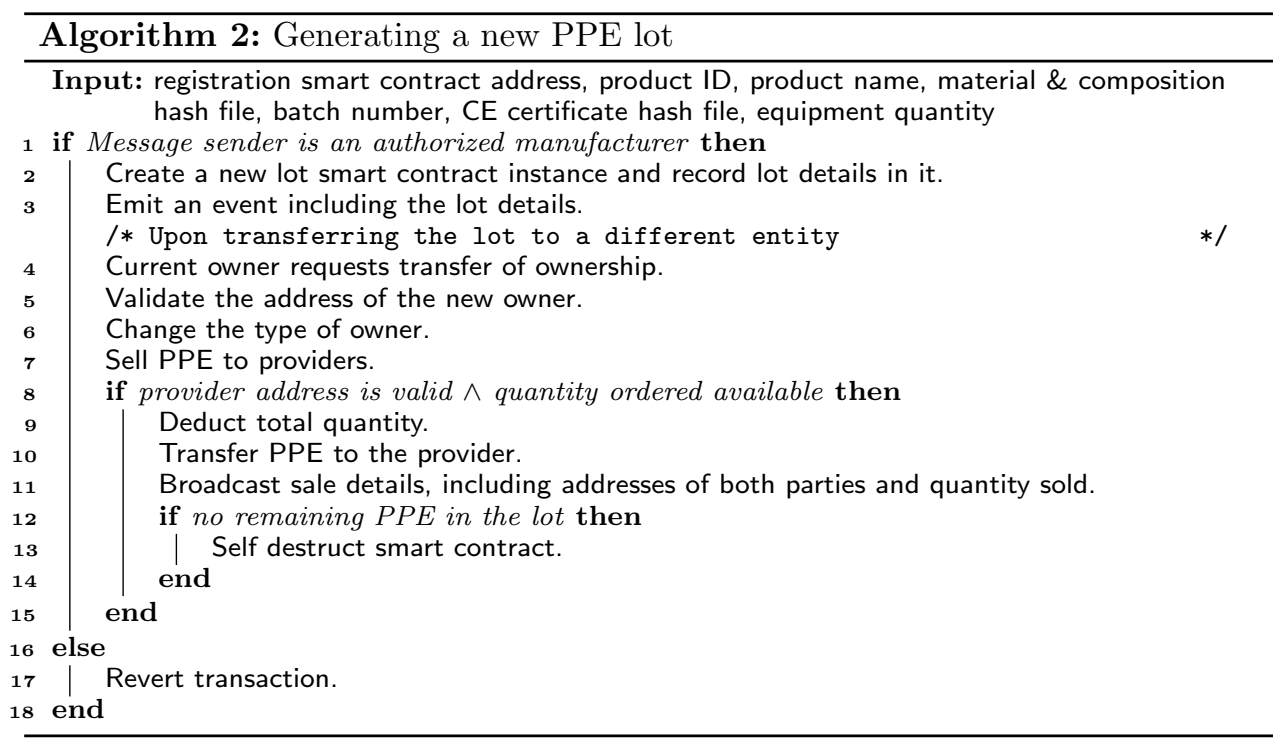

Alongside the confirmation of the order by the manufacturer, it also generates a lot smart contract as explained in algorithm 2. This smart contract communicates with the registration smart contract to verify that only approved manufacturers can generate these smart contracts. The manufacturer stores the details of the lot in its designated smart contract. These include information about the product, its certification, the materials used, and the number of items. In addition to confirm the order delivery as described in algorithm 1, the current owner also transfers ownership of the lot in the lot smart contract. This is done upon the delivery of the lot to every single entity until it reaches the wholesaler that ordered it. The smart contract validates the identity of each member before transferring ownership to it. It also automatically detects the role of the owner through its EA. When the wholesaler wants to dispense parts of the PPE to different providers (hospitals, pharmacies, healthcare centers, etc.), the smart contract deducts the sold quantity from the available stock, and the items are shipped to the provider. Subsequently, an event is triggered, announcing the details of the sale. Finally, the smart contract self destructs when the lot is exhausted.

\section{Testing and Validation}

This section provides a concise explanation of the testing strategy to verify and validate the solution developed. Remix IDE was utilized to test the various smart 
contracts. This IDE provides a virtual development, deployment, and testing platform to evaluate Ethereum smart contracts. Transactions are explored via Remix that include transaction source, input parameters, outputs, execution costs, and events triggered with their details. Debugging and correcting run-time errors is made easier with exceptions clearly displayed for developers. These errors are either enforced by the blockchain, such as gas limits, or implemented manually in the smart contract, such as incorrect addresses.

The registration and order management smart contracts are deployed once and are given unique addresses by the IDE. These addresses are constant and not like lot smart contracts that are generated dynamically by PPE manufacturers. To test the implemented solution, the different stakeholders are registered, and a test supply chain was implemented with different scenarios. Remix IDE provides a list of random Ethereum addresses that were used to enroll different entities through the registration smart contract. The distributor then sends an order request to the order management smart contract. The smart contract verifies the order and announces it so that the manufacturer can view it as it can be seen in fig. 7 . The event shown consists of the order ID, the manufacturer and distributor addresses, the product ID, and the quantity ordered. The smart contract was tested for approving and rejecting the order.

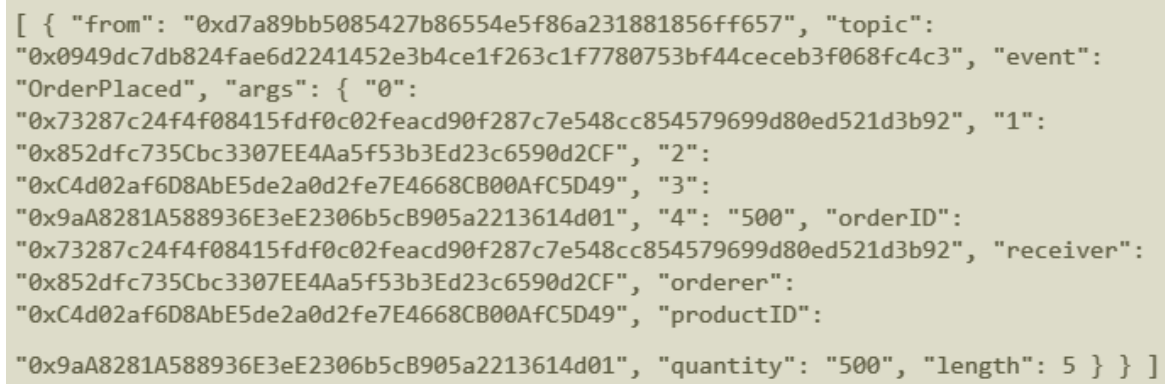

Figure 7 Event showing the placement of a new PPE order.

In the case of approval by the manufacturer, it informs the smart contract and proceeds to prepare the PPE lot for transfer. A new lot smart contract is also created. This smart contract holds the details of the lot, as shown in fig. 8. This smart contract is only allowed to be generated by approved manufacturers, which is confirmed by the registration smart contract. Once the distributor acquires the lot, it can sell it to wholesalers that dispense it to retailers and PPE providers, including hospitals and pharmacies.

Selling PPE to independent entities that use them or re-sells them is done through the lot smart contract as well. The smart contract first verifies the address of the provider. If the registration smart contract approves the EA, the lot smart contract deducts the amount ordered by the provider. If the available inventory in that lot is insufficient to meet the sale requirement, the smart contract generates an error, that can be seen in the logs of Remix. Fig. 9 shows the exception thrown when upon the event of stock shortage. 


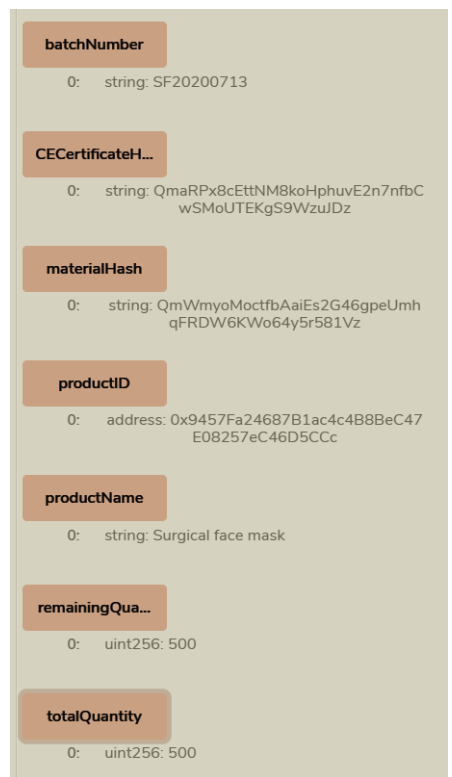

Figure 8 Lot details after being delivered.

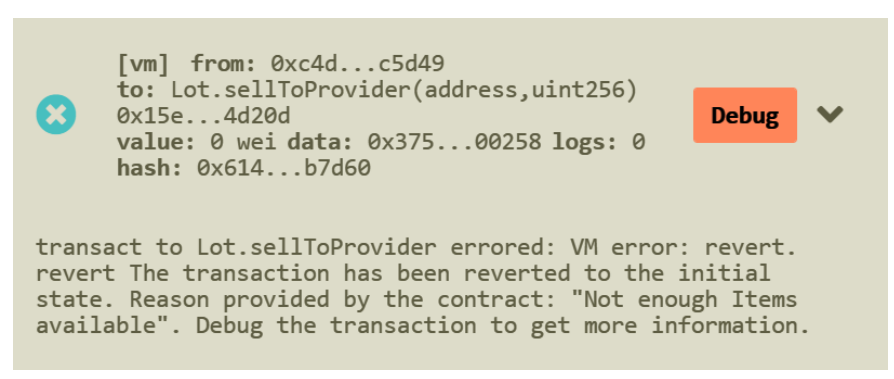

Figure 9 Exception message proving that the available stock is less than the requested items.

Finally, all available items in the lot are sold to retailers and providers. Therefore, the current lot smart contract is not useful and has no purpose. Therefore, it self destructs and is not accessible anymore. The previous events are still available for backtracking previous transactions. But no new transactions are possible. In Remix, it can be seen that the execution cost becomes zero has no functions executed by the smart contract, as shown in fig. 10.

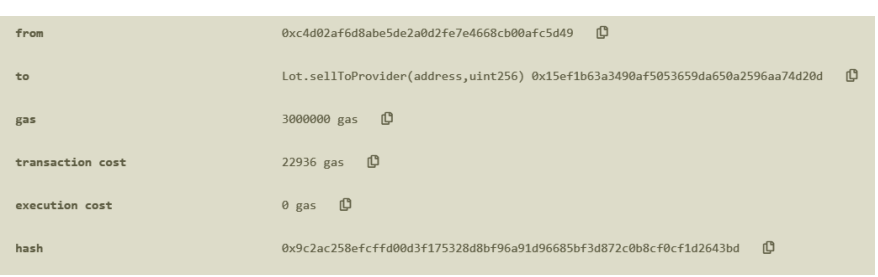

Figure 10 Execution gas cost hits zero after smart contract self destructs. 


\section{DISCUSSION}

In our paper, we have implemented a blockchain-based solution for tracking PPE, especially during pandemics. Our proposed solution captures the main operations that take place in PPE supply chains. We discuss the cost and security analysis of the proposed system.

Table 1 Gas cost of Ethereum functions in USD

\begin{tabular}{|c|c|c|c|c|c|}
\hline Method name & $\begin{array}{c}\text { Transac- } \\
\text { tion gas } \\
\text { cost }\end{array}$ & $\begin{array}{c}\text { Execution } \\
\text { gas cost }\end{array}$ & $\begin{array}{c}\text { Slow } \\
\text { execution } \\
\text { (USD) }\end{array}$ & $\begin{array}{c}\text { Average } \\
\text { execution } \\
\text { (USD) }\end{array}$ & $\begin{array}{c}\text { Fast } \\
\text { execution } \\
\text { (USD) }\end{array}$ \\
\hline registerManufacturer & 45709 & 23029 & 1.02822 & 1.09619 & 1.17268 \\
registerDistributor & 45754 & 23074 & 1.03025 & 1.09833 & 1.17497 \\
registerWholeSaler & 45775 & 23095 & 1.03117 & 1.09936 & 1.17604 \\
registerProvider & 45624 & 23008 & 1.0273 & 1.09519 & 1.17161 \\
transferOwnership & 55866 & 33186 & 1.48172 & 1.57969 & 1.68991 \\
sellioprovider & 39843 & 16971 & 0.75774 & 0.80785 & 0.8642 \\
placeOrder & 119230 & 94950 & 4.23944 & 4.51973 & 4.83504 \\
confirmorder & 51987 & 28347 & 1.26567 & 1.34936 & 1.44349 \\
confirmReceived & 35436 & 11988 & 0.53523 & 0.57066 & 0.61044 \\
\hline
\end{tabular}

\subsection{Cost Analysis}

Ethereum smart contract functions spend gas when triggered. The gas cost of a function is contingent on the function in the smart contract comprising its inputs, outputs, size of the Solidity code, and its complexity. These form the execution gas cost of the transaction. In addition, the cost of actually sending the transaction to the blockchain is added to the execution gas cost to make up the transaction gas cost. The actual price paid by the Ethereum client depends on the gas price that it sets. The gas price chosen by the client is multiplied by the gas price of the transaction to compute the number of ethers to be paid. Having said that, higher gas prices guarantee faster execution of the transaction as it would be more compelling to miners for higher rewards. Miners have little interest in mining transactions with low gas prices and would hardly pick them.

Table 1 presents the various methods in all the aforementioned smart contracts with the transaction and execution cost in gas, as reported by the IDE. To improve readability, the gas costs are converted to fiat currency (USD). As mentioned earlier, the actual cost of the transaction depends on the gas price of the Ethereum client. The cost analysis table includes three conversion rates, as mentioned in [16] on September 10th, 2020, at the time of this writing. Moreover, 1 Ether is currently worth $\$ 373.33$ on the same day. The three rates of conversion mentioned slow execution that costs 121 Gwei, or $\$ 0.000045$ per gas, average execution that costs 129 Gwei, or $\$ 0.000048$ per gas, and fast execution that costs 128 Gwei, or $\$ 0.000051$ per gas. With the exception of the placeOrder method, the cost of all function is less than $\$ 1.5$ each for slow execution, less than $\$ 1.6$ each for average execution, and less than $\$ 1.7$ each for fast execution. Placing orders cost a lot of gas due to the generation of a random order ID dynamically. This analysis justifies the use of Ethereum smart contracts for ordering and management of PPE.

\subsection{Security Analysis}

In this section, we discuss key properties of the proposed blockchain PPE tracking solution in addressing major security and privacy concerns related to confidentiality, data integrity, availability, non-repudiation, and vulnerability to cyberattacks. 
- Confidentiality: With respect to the fundamental features of blockchain, it use pseudonyms to protect identity of the participants ensuring their privacy. This, promotes transparency as all stakeholders in the supply chain would be aware of the activities occurring at any point in time as valid transactions are time-stamped and stored in the blockchain. Moreover, supply chain visibility is crucial to avoid making order errors. For instance, all manufacturers, distributors, and wholesalers are referred to by their EAs in the proposed system, and therefore, their identities remain protected.

- Data integrity: Integrity in a blockchain-based system means that data recorded in the network is accurate and is not allowed to be modified or deleted by anyone in the network. Moreover, data integrity is maintained as valid transactions in a block are secured using cryptographic mechanisms. This is possible as any order placed by a distributor or a wholesaler within the proposed system gets stored as a valid transaction in the ledger, which becomes immutable and cannot be canceled or reversed.

- Availability: This refers to the capability of the blockchain system to withstand attacks and remain operational even in the occurrence of malicious code such as a denial of service (DoS) attack. This is possible due to the distributed nature of blockchain technology. Thus, all functionalities such as ordering lots, dispatching, transferring ownership, and selling items are available at all times, even if several entities in the blockchain network were down.

- Non-repudiation: The users registered in the blockchain network cannot disavow regarding sending or receiving a transaction. This is beneficial as wholesalers, distributors, and providers in the PPE supply chain network cannot deny that orders were made incorrectly. Neither can manufacturers deny receiving and approving a new lot request.

- Man-in-The-Middle Attacks: Our solution is based on the blockchain and therefore inherits its features. All transactions mined in the Ethereum network is digitally encrypted. Therefore, attackers cannot tamper with the transaction as they would need the private key to sign the transaction. Hence, miners would not accept the transaction and add it to their block. Therefore, the integrity of all the communication between different entities of the supply chain is preserved.

\subsection{Generalization}

Our proposed solution is designed to be generic enough that it can be applied to any product type in the supply chain other than PPE. This is because any supply chain operation consists of the same stakeholders, which are the manufacturer, distributor, wholesaler, and provider or retailer. Thus, the same framework can be applied to cater to the needs of any other product that requires traceability and efficient management. The proposed smart contract is suitable for any product or good that is transferred from one entity to the other.

However, the implementation of Ethereum smart contracts would be required to be made public so that stakeholders in the supply chain network would be able to view the smart contracts and get alerts during emergencies. Moreover, the smart contract, in particular, would require alteration as it currently holds data suitable 
for PPE. Thus, only minimal changes would be needed to customize the source code of the smart contract so that the entry data fields fulfill the requirements needed for the desired product.

\section{CONCLUSION}

In this paper, we discussed the influence of the COVID-19 pandemic in healthcare supply chain operations, particularly in tracking and managing PPE. Integrating blockchain technology in the supply chain can enhance the traceability of products while maintaining data integrity and provenance. It also promotes transparency among the stakeholders, thereby increasing communication and trust among themselves. The presented blockchain-based PPE tracking solution uses Ethereum smart contracts to manage orders and transactions in a decentralized network. Although our focus in this paper in on the PPE supply chains, the proposed system can be adapted to address traceability needs in other business domains. Using smart contracts can achieve verifiable automation, reduce information asymmetry and other inefficiencies in the supply chain processes. The evaluation, cost and security analysis of the proposed system has highlights its feasibility in a real-world environment, addressing traceability challenges in a secure and cost-effective manner. As future work, we propose to design and build decentralized applications to fully automate the PPE supply chain process for all supply chain stakeholders.

\section{Acknowledgement}

This publication is based upon work supported by Khalifa University of Science and Technology under Award No. CIRA-2019-001 and RCII-2019-002- Research Center for Digital Supply Chain and Operations Management.

\section{Author details}

${ }^{1}$ Department of Industrial and Systems Engineering, Khalifa University of Science and Technology, Abu Dhabi, UAE. ${ }^{2}$ Department of Electrical and Computer Engineering, Khalifa University of Science and Technology, Abu Dhabi, UAE. ${ }^{3}$ School of Computing and Engineering, University of West London, London, UK.

\section{References}

1. Alegado, S.: Global cost of coronavirus may reach $\$ 4.1$ trillion, adb says. Bloomberg (2020). [Accessed: 15 August 2020]

2. Park, C.-Y., Kim, K., Roth, S.: Global shortage of personal protective equipment amid covid-19: supply chains, bottlenecks, and policy implications (2020)

3. Armani, A.M., Hurt, D.E., Hwang, D., McCarthy, M.C., Scholtz, A.: Low-tech solutions for the covid-19 supply chain crisis. Nature Reviews Materials, 1-4 (2020)

4. Lagasse, J.: Healthcare industry is grappling with the emergence of counterfeit ppe in the covid-19 battle. Healthcare Finance (2020). [Accessed: 19 Aug 2020]

5. Rana, S.S.: Rise of counterfeit ppe in india amid covid-19. ICLG.com (2020). [Accessed: 19 Aug 2020]

6. Buterin, V., et al.: Ethereum: A next-generation smart contract and decentralized application platform. URL https://github. com/ethereum/wiki/wiki/\% 5BEnglish\% 5D-White-Paper 7 (2014)

7. Vosoughi, H.: Online markets of counterfeit products: a comparison between high-tech \& low-tech products (2020)

8. Thorbecke, C.: Amazon removes 1 million products for misleading claims, price gouging amid coronavirus outbreak. ABC News (2020). [Accessed: 28 Jul 2020]

9. Zheng, Z., Xie, S., Dai, H., Chen, X., Wang, H.: An overview of blockchain technology: Architecture, consensus, and future trends. In: 2017 IEEE International Congress on Big Data (BigData Congress), pp. 557-564 (2017). IEEE

10. Priyanka, S.S., Lokeswari, N., Amaravathi, K.: Blockchain-a potential solution for covid-19 pandemic economical crisis environment

11. Toyoda, K., Mathiopoulos, P.T., Sasase, I., Ohtsuki, T.: A novel blockchain-based product ownership management system (poms) for anti-counterfeits in the post supply chain. IEEE access 5, 17465-17477 (2017)

12. Malik, S., Kanhere, S.S., Jurdak, R.: Productchain: Scalable blockchain framework to support provenance in supply chains. In: 2018 IEEE 17th International Symposium on Network Computing and Applications (NCA), pp. 1-10 (2018). IEEE

13. Wang, S., Li, D., Zhang, Y., Chen, J.: Smart contract-based product traceability system in the supply chain scenario. IEEE Access 7, 115122-115133 (2019)

14. Parizi, R.M., Dehghantanha, A., et al.: Smart contract programming languages on blockchains: an empirical evaluation of usability and security. In: International Conference on Blockchain, pp. 75-91 (2018). Springer

15. Benet, J.: Ipfs-content addressed, versioned, p2p file system. arXiv preprint arXiv:1407.3561 (2014)

16. Eth gas station. [Accessed: 10 Sep 2020] 